Check for updates

Cite this: RSC Adv., 2018, 8, 8755

Received 25th January 2018

Accepted 13th February 2018

DOI: $10.1039 / c 8 r a 00787 j$

rsc.li/rsc-advances

\section{A highly active worm-like PtMo nanowire for the selective synthesis of dibenzylamines $\uparrow$}

\begin{abstract}
Shuanglong Lu, Pengyao Xu, Xueqin Cao and Hongwei Gu (DD*
Worm-like nanowires are among the most active nanomaterials. In this study, we report the synthesis of dibenzylamine (DBA) motifs from reductive amination of either aldehydes or nitriles catalyzed by entirely new worm-like PtMo nanowires (PtMo WNWs). Under the assistance of $\mathrm{H}_{2}$ gas, PtMo WNWs can be prepared in a facile manner, following which, their structure and composition are characterized by TEM, XRD, XPS, etc. Upon careful optimization of reaction parameters, the as-prepared PtMo WNWs work effectively in the activation of dihydrogen molecules, and both aldehydes and nitriles can be used as starting materials to fabricate DBAs under mild and green conditions. The reaction kinetics has been investigated, which reveals that the PtMo WNWs show superior activity in the conversion of imines into amines. This study provides a practical advancement in the preparation of amines. Moreover, the protocol reported herein is feasible for the synthesis of worm-like nanostructures with designed composition for various catalytic applications.
\end{abstract}

\section{Introduction}

Dibenzylamine (DBA) motifs are important compounds with extensive applications in rubber compounds, fine chemicals, corrosion inhibitors, and drug formulations; ${ }^{1-4}$ in this regard, the development of methods allowing the selective synthesis of DBA is of great significance in organic chemistry. Through significant research efforts made in this direction, synthetic routes, such as direct base-promoted mono- $N$-alkylation ${ }^{5}$ or alkylative amination, ${ }^{6,7}$ have been developed for the synthesis of DBA motifs. However, problems such as the use of expensive starting materials, tedious workup procedures, low selectivity, and formation of large amounts of wasteful salts still remain to be addressed.

Reductive amination is a highly versatile and robust method for various transformations involved in the $\mathrm{C}-\mathrm{N}$ bond construction..$^{8-10}$ It offers compelling advantages such as mild reaction conditions, inexpensive reagents, and a wide availability of substrates over other classical amine synthesis methods. ${ }^{11-13}$ Among various reduction amination processes, the activation of dihydrogen and the catalytic reduction of unsaturated organic molecules is a fundamentally promising

Key Laboratory of Organic Synthesis of Jiangsu Province, College of Chemistry, Chemical Engineering and Materials Science, Collaborative Innovation Center of Suzhou Nano Science and Technology, Soochow University, Suzhou, China 215123. E-mail: hongwei@suda.edu.cn

$\uparrow$ Electronic supplementary information (ESI) available: Experimental procedures, PXRD, EDS and XPS patterns for the PtMo WNWs, GC test for the reductive amination of $\mathrm{BzH}$ and $\mathrm{BzN}$, the influence of ammonia amount in the yield of DBAs, control experiments, NMR analysis for all amine products. See DOI: $10.1039 / \mathrm{c} 8 \mathrm{ra} 00787 \mathrm{j}$ green process for the production of amines. ${ }^{14-18}$ Conventional strategies, using Raney ${ }^{\circledR} \mathrm{Ni}$ or Tin compounds, suffer from low selectivity and poor stability. ${ }^{19}$ Alternatively, transition metalbased organometallic complexes have been proven to be efficient catalysts to cleave dihydrogen molecules ${ }^{20}$ but their complete removal from the reaction product presents a big concern when it comes to the production of pharmaceutical intermediates. Moreover, precise design and synthesis are required for organometallic catalysts. Recently, use of welldefined metal nanostructures in catalytic hydrogenation processes has become a rapidly growing area of research..$^{21-25}$ Somorjai and Yang reported the catalytic hydrogenation of pyrrole using platinum nanocrystals and found that the activity and selectivity were heavily dependent on the size and shape of the nanocrystals. ${ }^{26}$ Cao and his coworkers utilized gold-based nanostructures to activate dihydrogen molecules. ${ }^{27}$ Unsaturated organic compounds, such as quinolones, were hydrogenated under mild conditions.

Herein, we report entirely new worm-like PtMo nanowires (WNWs) prepared through facile co-reduction of Pt and Mo precursors under the assistance of $\mathrm{H}_{2}$ gas. The as-prepared nanowires work effectively in the activation of dihydrogen, following which, DBAs can be obtained from the reductive amination of either benzaldehyde (BzH) or benzonitriles (BzN) under mild and green reaction conditions (Scheme 1). Wormlike nanowires are among the most active nanostructures, which are widely studied in both electro-catalysis and organic transformation. ${ }^{28-30}$ The wavy structure and the incorporation of Mo are favorable to enhance the catalytic activity and durability, respectively. ${ }^{31}$ Furthermore, the newly developed strategy for PtMo WNWs simplifies the synthetic process for 


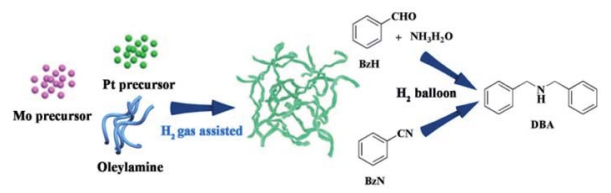

Scheme 1 Schematic of the synthesis of PtMo WNWs and its catalytic application in the DBA synthesis from reductive amination of either $\mathrm{BzH}$ or $\mathrm{BzN}$.

nanostructured catalysts, providing a much more practical pathway for catalytic applications.

\section{Experimental}

\section{Synthesis of worm-like PtMo nanowires}

To prepare worm-like PtMo wavy nanowires, a Pt precursor (platinum acetylacetonate, $0.125 \mathrm{mmol}, 49.2 \mathrm{mg}$ ) and a Mo precursor (molybdenyl acetylacetonate, $0.125 \mathrm{mmol}, 40.8 \mathrm{mg}$ ) were added to OAm $(31 \mathrm{mmol}, 10 \mathrm{~mL})$ in a three-necked flask, followed by stirring for $30 \mathrm{~min}$ at $100{ }^{\circ} \mathrm{C}$ under a $\mathrm{N}_{2}$ atmosphere to ensure that the precursors were dissolved thoroughly. The solution was cooled down to room temperature and transferred into an autoclave, which was then charged with $\mathrm{H}_{2}$ to 5 bar. The autoclave was heated to $165{ }^{\circ} \mathrm{C}$ and kept at this temperature for $2 \mathrm{~h}$ under stirring. The resulting black colloidal products were obtained by centrifugation and washed several times with ethanol and chloroform.

\section{Material characterization}

The morphologies of the worm-like PtMo nanowires were determined by a TEM (Tecnai G220, FEI, USA) equipped with a Gatan CCD794 camera operated at $200 \mathrm{kV}$. The HAADF-STEM, elemental mapping, HR-TEM, and EDX of a single nanohybrid were carried out using a Tecnai G2 F20 instrument at an accelerating voltage of $200 \mathrm{kV}$. The XRD pattern was obtained using an X'Pert-Pro MPD diffractometer (the Netherlands PANalytical) with a Cu K $\alpha$ X-ray source $(\lambda=1.540598 \AA)$.

\section{General procedure for the reductive amination of aldehydes} and nitriles

Reactants, solvents, and catalysts were added to the reaction tube that was then sealed. The reaction tube was evacuated thrice and then flushed with hydrogen at a certain temperature under an atmosphere of hydrogen. The resulting product mixtures were analyzed by GC (VARIAN CP-3800 GC, HP-5 capillary column, FID detector) and GC-MS (VARIAN 450-GC \& VARIAN 240-GC) equipped with a CP8944 capillary column (30 $\mathrm{m} \times 0.25 \mathrm{~mm}$ ). Products were purified by flash chromatography and characterized by ${ }^{1} \mathrm{H}$ NMR and ${ }^{13} \mathrm{C}$ NMR spectroscopies.

\section{Results and discussion}

The PtMo WNWs were prepared through co-reduction of Pt and Mo metallic precursors in oleylamine (OAm) by heating to $165{ }^{\circ} \mathrm{C}$ in an autoclave under $\mathrm{H}_{2}$ gas. The detailed procedure is provided in the experimental section.
Fig. 1A and $\mathrm{B}$ show the representative transmission electron microscopy (TEM) and high-angle annular dark-field scanning transmission electron microscopy (HAADF-STEM) images of the as-prepared PtMo WNWs, respectively, that are highly uniform with nearly $100 \%$ nanowire morphology. As can be seen, these nanowires are highly dispersed with a narrow size distribution. The average diameter of the product is around $4.8 \mathrm{~nm}$, and the length is $100 \mathrm{~nm}$. Impressively, the high-resolution TEM (HRTEM) image shown in Fig. 1C reveals that these nanowires are composed of multiple crystalline domains, and the observed fringes are from the $\{111\}$ planes. The continuous lattice fringes from the inner to the outer areas of PtMo WNWs without an obvious phase segregation demonstrate high amalgamation between Pt and Mo atoms. Moreover, the selected-area electron diffraction (SEAD) pattern shows a typical metallic facecentered cubic (fcc) structure, which is in agreement with the powder X-ray diffraction (PXRD) pattern (Fig. S1†). Intriguingly, there are no single peaks for Mo or Pt in the XRD patterns; this implies the purity of the PtMo alloy without undesired phases. The atomic ratio of $\mathrm{Pt} / \mathrm{Mo}$ is $93.56 / 6.43$, determined by an energy dispersive spectrometer (EDS) (Fig. S2 $\dagger$ ). The observed low content of Mo is due to the high stability of Mo precursors against inferior reductants, such as $\mathrm{H}_{2}$, resulting in incomplete reduction of Mo precursors, which is consistent with previous studies. ${ }^{31,32}$ The binding energy of $\mathrm{Pt} 4 \mathrm{f}$ is around 71.3, which is determined by the XPS analysis shown in Fig. S3, $\uparrow$ and it shifts to a higher value relative to that of pure Pt NCs (70.9) due to the alteration of the Pt electronic structure with Mo atoms that downshifts the d-band center of Pt. The formation of the PtMo alloy is further investigated by the elemental mapping analysis (Fig. 1D). This reveals that Pt and Mo are well-distributed throughout the interior and exterior domains.

To demonstrate the high catalytic activity of these PtMo WNWs in the preparation of DBAs, BzH was selected as the substrate for the optimization of reaction conditions. Table 1 shows the reductive amination of $\mathrm{BzH}$ with aqueous ammonia as the economical $\mathrm{N}$ source.

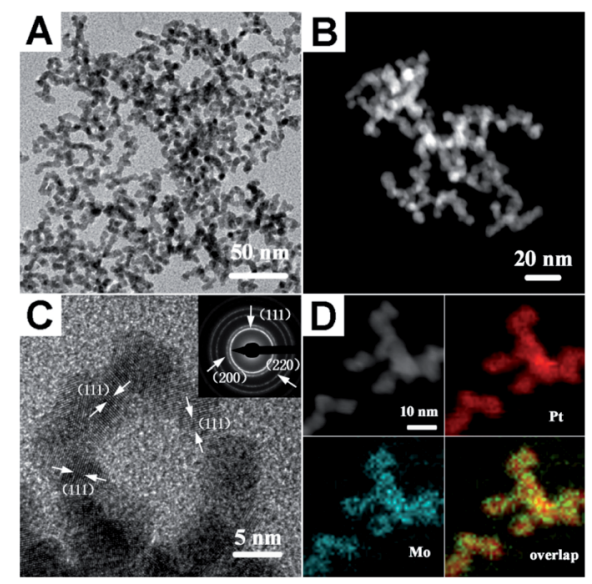

Fig. 1 (A) Representative TEM image; (B) HAADF-STEM image; (C) HRTEM image, the inset shows the SAED pattern; and (D) highmagnification HAADF-STEM image and the corresponding elemental mapping of the worm-like PtMo nanowires. 
Table 1 Optimization of reaction conditions using benzaldehyde as a substrate ${ }^{a}$

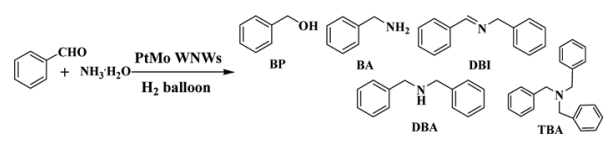

\begin{tabular}{lllllllll}
\hline & & & \multicolumn{7}{c}{ Yield $^{b}(\%)$} \\
\cline { 6 - 9 } Entry & Solvent & $T /{ }^{\circ} \mathrm{C}$ & Conv. $^{b}(\%)$ & BP & DBI & DBA & TBA \\
\hline \multirow{2nnnnyyyyy}{*}{} & Methanol & 60 & 100 & 3.2 & 19.1 & 70.2 & - \\
2 & $n$-Heptane & 80 & 100 & 3.1 & - & 91.4 & 5.5 \\
3 & Water & 80 & 100 & 66.1 & 3.6 & 30.3 & - \\
4 & Ethanol & 80 & 98.8 & 26.2 & 3.2 & 40.9 & 28.6 \\
5 & DMF & 80 & 96.4 & 4.2 & 58.5 & 33.7 & - \\
6 & 1,4-Dioxane & 100 & 64.8 & 54.2 & 1.2 & 4.5 & 4.9 \\
7 & Toluene & 100 & 100 & 16.7 & - & - & 83.3 \\
8 & $p$-Xylene & 100 & 100 & 3.1 & - & 92.8 & 1.7 \\
9 & $o$-Xylene & 100 & 100 & 2.4 & - & 92.4 & 2.8 \\
10 & $m$-Xylene & 100 & 100 & 0.5 & 0.8 & 96.1 & 0.8 \\
11 & $m$-Xylene & 80 & 100 & 16.6 & 10.1 & 67.0 & - \\
12 & $m$-Xylene & 60 & 95.2 & 17.5 & 57.4 & 18.3 & - \\
13 & $m$-Xylene & 40 & 97.2 & 9.4 & 74.1 & 13.8 & -
\end{tabular}

${ }^{a}$ Reaction conditions: $\mathrm{BzH}(1.0 \mathrm{mmol}), 25 \%$ aqueous ammonia (2 equiv.), and solvent ( $2 \mathrm{~mL}$ ) at 1 bar $\mathrm{H}_{2}$ with $0.5 \mathrm{~mol} \%$ PtMo WNWs for 24 h. ${ }^{b}$ GC yield.

Through GC-MS analysis, we have obtained the idea that besides DBA, side products, such as benzylalcohol (BP) benzylamine (BA), dibenzylimine (DBI), and tribenzylamine (TBA), can be detected (Fig. S4 $\dagger$ ). Various solvents were first screened at the corresponding temperatures by employing a $\mathrm{H}_{2}$ gas balloon (Table 1, entries 1-10). Polar solvents (entry 1, 3-6) seem to be unsuitable to improve the selectivity in the reductive amination of $\mathrm{BzH}$ via the PtMo catalysis. When $n$-heptane was used as a solvent (entry 2), the yield of DBA increased to $91.4 \%$, a relatively satisfactory result. Interestingly, we obtained TBA as the main product when toluene was used (entry 7). The best solvent in our screening was found to be xylene, and the yields of $92.4 \%, 96.1 \%$, and $92.8 \%$ were obtained for DBA in $o, m$, and $p$-xylene, respectively (entry 8-10). Based on these results, $m$ xylene was chosen as the best solvent for the following investigation on the temperature influence. Obviously, with a decrease in the reaction temperature, a corresponding steady decrease in the BDA yield was found, as presented in Table 1 (entry 10-13). The molar ratio between $\mathrm{BzH}$ and ammonia was also vital to the reduction activity. When ammonia was absent, BP was the major product. A higher or lower concentration of ammonia was more or less not favorable for DBA formation (Table S1 $\dagger$ ). All these observations indicate that PtMo WNWs present a good result for DBA synthesis.

Due to the highly efficient catalytic performance of PtMo WNWs in the reductive animation of the aldehyde under $\mathrm{H}_{2}$ gas, we examined the catalytic capability of PtMo in the reductive hydrogenation of nitriles (Table 2). Nitriles, which are bioavailable in nature, are a promising feasible alternative to fabricate DBAs because no additional $\mathrm{N}$ sources are required. ${ }^{33,34}$ However, how to enhance the selectivity for the reduction of nitriles still remains a challenge. ${ }^{35-37}$

As indicated in Table 2, contrary to the situation when BzH was used, except heptane, most solvents were suitable for the reductive amination of BzN (Table 2, entries 1-9). Among them, ethanol was found to be the best solvent, yielding 95.4\% DBA at $80{ }^{\circ} \mathrm{C}$ (Fig. S5 $\dagger$ ) after we carefully evaluated the temperature influence. In the control experiment, no reduced products were observed in the absence of either a catalyst or $\mathrm{H}_{2}$ gas (Table $\mathrm{S} 2, \uparrow$ entry 1,2). Moreover, commercial Pt/C (20\%) was used in the reductive amination of $\mathrm{BzH}$ or $\mathrm{BzN}$ for comparison (Table $\mathrm{S} 2, \dagger$ entry 3,4). Obviously, low selectivity was observed, and TBA emerged as the main side product. Pt nanoparticles and Pt nanorods with a smooth surface were also synthesized and applied in the reduction of nitriles for comparison (Table S3 $\dagger$ ). Apparently, worm-like PtMo nanowires show much better performance. The enhancement in the catalytic activity could be attributed to their wavy structures, which could provide numerous structural defects and grain boundaries. It has been previously suggested that the surface defects can serve as possible channels for incorporating small molecules into the surface region. ${ }^{38-40}$ The incorporation of Mo into Pt enhances the activity and stability of Pt, which has been comprehensively investigated in previous studies. ${ }^{41}$ Moreover, the synergistic electronic effect between two metals may have important role in the catalytic reaction. ${ }^{42}$ Hydrogen on the surface of Pt sites can migrate over to the Mo species, liberating the Pt active sites. ${ }^{31}$

Real-time GC measurement was used to track the kinetic processes of the transformation. Fig. $2 \mathrm{~A}$ and $\mathrm{C}$ show the timeconversion plots when $\mathrm{BzH}$ and $\mathrm{BzN}$ are used as the starting reactants, respectively. As can be seen, with the consumption of the either starting substrate, the only intermediate that can be detected in common is DBI. However, they differ from each other in terms of the concentration of the DBI formed. For reduction amination using $\mathrm{BzH}$, the concentration of DBI

Table 2 Optimization of the reaction conditions using benzonitrile as a substrate ${ }^{a}$

\begin{tabular}{|c|c|c|c|c|c|c|}
\hline \multirow[b]{2}{*}{ Entry } & \multirow[b]{2}{*}{ Solvent } & \multirow[b]{2}{*}{$T /{ }^{\circ} \mathrm{C}$} & \multirow[b]{2}{*}{ Conv. $^{b}(\%)$} & \multicolumn{3}{|c|}{ Conv. $^{b}(\%)$} \\
\hline & & & & BA & DBA & DBI \\
\hline 1 & Methanol & 40 & 94.9 & - & 91.0 & 1.5 \\
\hline 2 & $n$-Heptane & 80 & 80.6 & - & 56.8 & 23.6 \\
\hline 3 & 1,4-Dioxane & 100 & 93.1 & 4.5 & 84.5 & 7.4 \\
\hline 4 & Toluene & 100 & 98.2 & 2.3 & 91.0 & 4.9 \\
\hline 5 & Water & 100 & 98.4 & 4.5 & 85.0 & - \\
\hline 6 & $p$-Xylene & 100 & 98.5 & 3.1 & 91.3 & - \\
\hline 7 & $m$-Xylene & 100 & 100 & 1.4 & 93.5 & 5.1 \\
\hline 8 & $o$-Xylene & 100 & 100 & - & 92.4 & 7.6 \\
\hline 9 & Ethanol & 40 & 98.0 & 4.0 & 86.3 & 7.7 \\
\hline 10 & Ethanol & 60 & 100 & 1.5 & 94.3 & 4.2 \\
\hline 11 & Ethanol & 80 & 100 & - & 95.4 & 4.6 \\
\hline
\end{tabular}

${ }^{a}$ Reaction conditions: BzN $(1.0 \mathrm{mmol})$ and solvent $(2 \mathrm{~mL})$ at 1 bar $\mathrm{H}_{2}$ with 0.5 mol\% PtMo WNWs for 24 h. ${ }^{b}$ GC yield. 


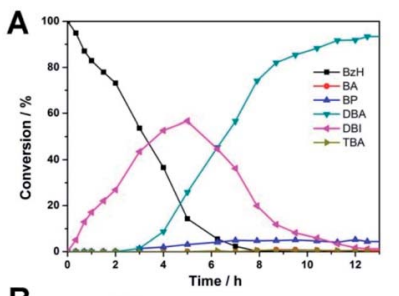

B
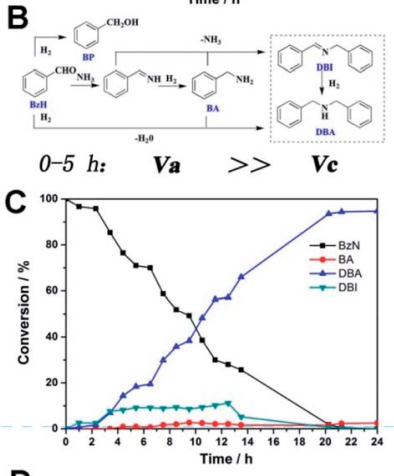

D

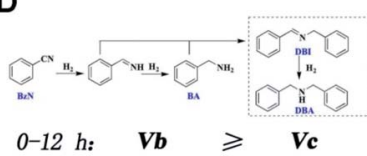

Fig. 2 (A) and (B) Time-conversion plot and proposed mechanism for the DBA formation using $\mathrm{BzH}$ as the starting reactant under the optimized reaction conditions; (C) and (D) with $\mathrm{BzN}$ as the starting reactant.

reached a peak value of $56.7 \%$ after 5 hours, whereas the amount of DBI, as shown in Fig. 2C, for BzN time-conversion remained stable at a steady level below $10.0 \%$.

Based on these observations and our previous studies, we have proposed mechanisms for the two separate systems (Fig. 2B and D). We defined the reaction velocity of BzH to DBI as $V_{\mathrm{a}}$, BzN to DBI as $V_{\mathrm{b}}$, and DBI to DBA as $V_{\mathrm{c}}$. Apparently, as shown in Fig. 2A, the intermediate DBI formed cannot be successively consumed to form DBA; this leads to the accumulation of DBI in the mixture. $V_{\mathrm{a}}$ is far greater than $V_{\mathrm{c}}$.

It is reasonable that the two proposed pathways are available for BzH to form DBI, which are coupling of BA with phenylmethanimine or BzH itself (Fig. 2B). On the other hand, for BzN, only one pathway is available, and most of the time, the formation and consumption of BDI reaches a dynamic equilibrium; $V_{\mathrm{a}}$ is slightly greater or equal to $V_{\mathrm{c}}$. Moreover, trace amounts of side products, such as BA, BP, and TBA, can be detected in the BzH system, whereas only BA can be detected in the BzN system. Compared with our previous studies, ${ }^{21,22}$ it is highly noteworthy that $V_{\mathrm{c}}$ is significantly improved; this demonstrates that PtMo WNWs are much more efficient in the conversion of imines into amines. The potential application of PtMo WNWs is ongoing in our group, and we will communicate these results in due course.

To determine the scope of these two pathways in the synthesis of DBA motifs, typical substrates of aldehyde and nitrile were chosen to be subjected to their optimized reaction conditions. As shown in Table 3, irrespective of the electronic nature of the substituent, good to excellent yields of DBAs can
Table 3 Formation of DBA motifs with different substrates through various pathways ${ }^{a, b}$

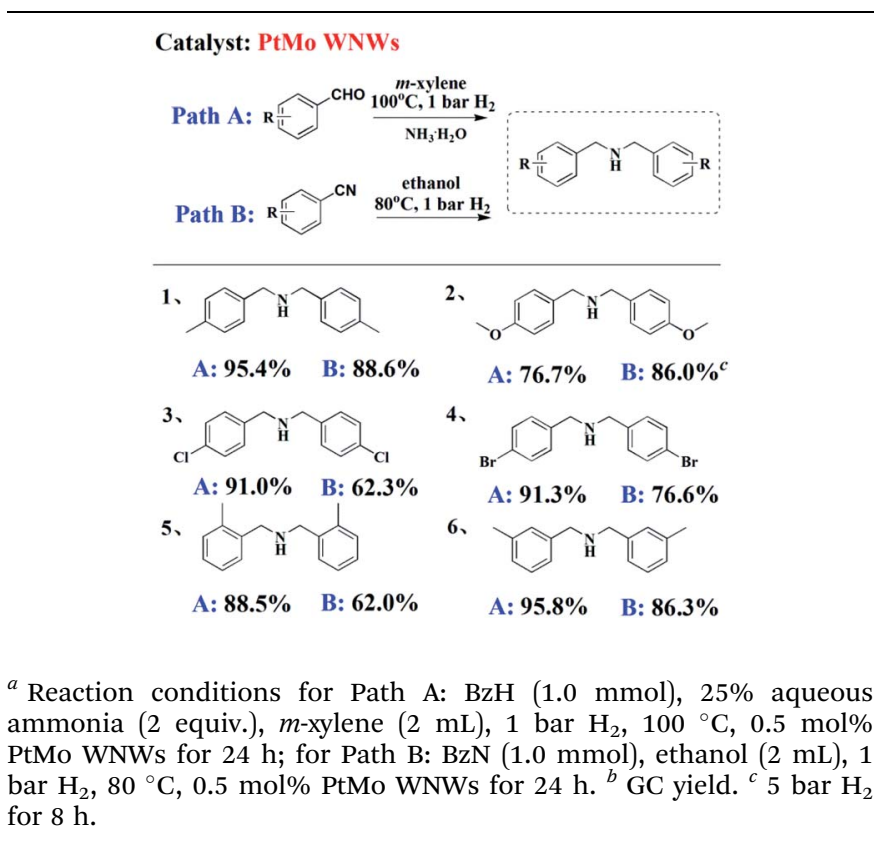

be obtained from the reductive amination of either $\mathrm{BzH}$ or BzN (entry 1-4). Upon comparing ortho-, meta-, and para-substituted $\mathrm{BzH}$ or BzH (entry 1, 5 and 6), steric hindrance effect was proven to exert little influence on the reaction yield. Notably, the use of higher pressures led to an increase in the yield of DBAs and shortened the reaction time for some substrates (entry $2 \mathrm{~B}$ ).

Finally, we investigated the upscale catalysis performance and recyclability of PtMo WNWs. As shown in Fig. 3, a yield of 94.6\% for DBA was obtained when 5-fold BzH was subjected to the optimized reaction conditions. An equally satisfactory result was also found in the situation of BzN. The PtMo WNWs before and after the reaction were characterized by TEM images, as shown in Fig. 3. No significant changes were observed. Impressively, there was also no discernible loss in activity after several runs (Fig. S6†).

The excellent catalytic activity and stability of worm-like PtMo nanowires can be attributed to the following three

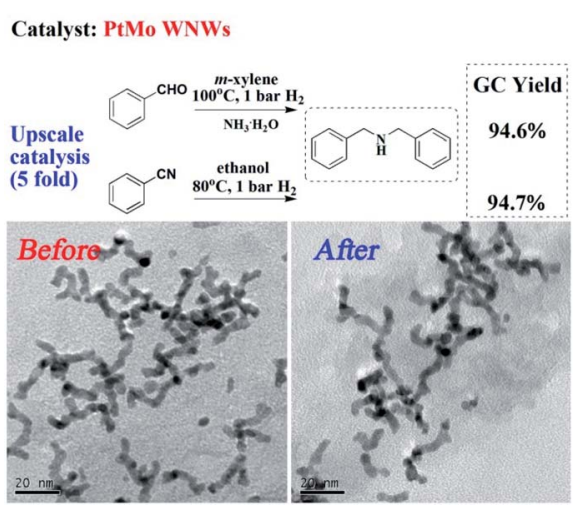

Fig. 3 Upscale catalysis performance and recycling of the catalyst. 
factors: (1) the worm-like structure provides numerous catalytic sites as compared to a smooth structure. (2) The incorporation of Mo into Pt can enhance the activity and stability of Pt, which has been comprehensively investigated in previous studies. ${ }^{\mathbf{4 1}}$ Moreover, the synergistic electronic effect between two metals may have an important role in the catalytic reaction. ${ }^{42}$ Hydrogen on the surface of Pt sites can migrate over to the Mo species, liberating the Pt active sites. ${ }^{\mathbf{3 1}}$ (3) The nanowire network is less susceptible to aggregation and dissolution.

\section{Conclusions}

In conclusion, a facile method was developed for the preparation of entirely new PtMo WNWs. The as-obtained worm-like nanowires work effectively in the activation of dihydrogen, through which, DBAs are successfully synthesized by the reductive amination of either $\mathrm{BzH}$ or $\mathrm{BzN}$. After careful evaluation of the kinetic processes, we found that the catalyst was much more active in the conversion of imines into amines due to its composition and morphological effects. Moreover, it can be readily recovered, and the catalytic system can be easily scaled up. Further study of this catalytic process and potential applications of PtMo WNWs are under investigation in our laboratory.

\section{Conflicts of interest}

There are no conflicts to declare.

\section{Acknowledgements}

This work was financially supported by the National Natural Science Foundation of China (NSFC) (Grant No. 21373006). The project was funded by the Priority Academic Program Development of Jiangsu Higher Education Institutions (PAPD).

\section{References}

1 T. Henkel, R. M. Brunne, H. Muller and F. Reichel, Angew. Chem., Int. Ed., 1999, 38, 643-647.

2 M. Berger, B. Albrecht, A. Berces, P. Ettmayer, W. Neruda and M. Woisetschläger, J. Med. Chem., 2001, 44, 3031-3038.

3 C. Su, R. Tandiana, J. Balapanuru, W. Tang, K. Pareek, C. T. Nai, T. Hayashi and K. P. Loh, J. Am. Chem. Soc., 2015, 137, 685-690.

4 P. P. Zhao, X. F. Zhou, J. J. Dai and H. J. Xu, Org. Biomol. Chem., 2014, 12, 9092-9096.

5 M. H. S. A. Hamid, P. A. Slatford and J. M. J. Williams, Adv. Synth. Catal., 2007, 349, 1555-1575.

6 O. Saidi, A. J. Blacker, M. M. Farah, S. P. Marsden and J. M. Williams, Angew. Chem., Int. Ed., 2009, 48, 7375-7378.

7 T. T. Dang, B. Ramalingam, S. P. Shan and A. M. Seayad, ACS Catal., 2013, 3, 2536-2540.

8 G. Liang, A. Wang, L. Li, G. Xu, N. Yan and T. Zhang, Angew. Chem., Int. Ed., 2017, 56, 3050-3054.

9 T. Komanoya, T. Kinemura, Y. Kita, K. Kamata and M. Hara, J. Am. Chem. Soc., 2017, 139, 11493-11499.
10 G. Gao, P. Sun, Y. Li, F. Wang, Z. Zhao, Y. Qin and F. Li, ACS Catal., 2017, 7, 4927-4935.

11 M. Chatterjee, T. Ishizaka and H. Kawanami, Green Chem., 2016, 18, 487-496.

12 K. G. Andrews, D. M. Summers, L. J. Donnelly and R. M. Denton, Chem. Commun., 2016, 52, 1855-1858.

13 C. Wang, A. Pettman, J. Basca and J. Xiao, Angew. Chem., Int. Ed., 2010, 49, 7548-7552.

14 P. Jochmann and D. W. Stephan, Chem.-Eur. J., 2014, 20, 8370-8378.

15 V. Sumerin, F. Schulz, M. Atsumi, C. Wang, M. Nieger, M. Leskelä, T. Repo, P. Pyykkö and B. Rieger, J. Am. Chem. Soc., 2008, 130, 14117-14119.

16 Y. Li, I. Sorribes, T. Yan, K. Junge and M. Beller, Angew. Chem., Int. Ed., 2013, 52, 12156-12160.

17 G. Zhang, B. L. Scott and S. K. Hanson, Angew. Chem., Int. Ed., 2012, 51, 12102-12106.

18 Y. Misumi, H. Seino and Y. Mizobe, J. Am. Chem. Soc., 2009, 131, 14636-14637.

19 S. Zinovyev, A. Perosa, S. Yufit and P. Tundo, J. Catal., 2002, 211, 347-354.

20 P. Jochmann and D. W. Stephan, Angew. Chem., Int. Ed., 2013, 52, 9831-9835.

21 M. Pan, A. J. Brush, Z. D. Pozun, H. C. Ham, W. Y. Yu, G. Henkelman, G. S. Hwang and C. B. Mullins, Chem. Soc. Rev., 2013, 42, 5002-5013.

22 J. B. Wu, P. P. Li, Y. T. Pan, S. Warren, X. Yin and H. Yang, Chem. Soc. Rev., 2012, 41, 8066-8074.

23 S. Lu, J. Wang, X. Cao, X. Li and H. Gu, Chem. Commun., 2014, 50, 3512-3515.

24 F. Qi, L. Hu, S. Lu, X. Cao and H. Gu, Chem. Commun., 2012, 48, 9631-9633.

25 L. Hu, X. Cao, L. Chen, J. Zheng, J. Lu, X. Sun and H. Gu, Chem. Commun., 2012, 48, 3445-3447.

26 C.-K. Tsung, J. N. Kuhn, W. Huang, C. Aliaga, L.-I. Hung, G. A. Somorjai and P. Yang, J. Am. Chem. Soc., 2009, 131, 5816-5822.

27 X. Liu, L. He, Y.-M. Liu and Y. Cao, Acc. Chem. Res., 2014, 47, 793-804.

28 X. Yu, D. Wang, Q. Peng and Y. Li, Chem.-Eur. J., 2013, 19, 233-239.

29 W. Liu, P. Rodriguez, L. Borchardt, A. Foelske, J. Yuan, A. K. Herrmann, D. Geiger, Z. Zheng, S. Kaskel, N. Gaponik, R. Kotz, T. J. Schmidt and A. Eychmuller, Angew. Chem., Int. Ed., 2013, 52, 9849-9852.

30 L. Hu, X. Cao, L. Shi, F. Qi, Z. Guo, J. Lu and H. Gu, Org. Lett., 2011, 13, 5640-5643.

31 E. Lee, A. Murthy and A. Manthiram, Electrochim. Acta, 2011, 56, 1611-1618.

32 Z. Liu, J. E. Hu, Q. Wang, K. Gaskell, A. I. Frenkel, G. S. Jackson and B. Eichhorn, J. Am. Chem. Soc., 2009, 131, 6924-6925.

33 S. Lu, C. Li, J. Wang, Y. Pan, X. Cao and H. Gu, Chem. Commun., 2014, 50, 11110-11113.

34 J. J. W. Bakker, A. G. v. d. Neut, M. T. Kreutzer, J. A. Moulijn and F. Kapteijn, J. Catal., 2010, 274, 176-191. 
35 A. Mukherjee, D. Srimani, S. Chakraborty, Y. Ben-David and D. Milstein, J. Am. Chem. Soc., 2015, 137, 8888-8891.

36 G. Clavel, V. Molinari, A. Kraupner and C. Giordano, Chem.Eur. J., 2014, 20, 9018-9023.

37 J.-H. Choi and M. H. G. Prechtl, ChemCatChem, 2015, 7, 1023-1028.

38 X. Huang, Z. Zhao, Y. Chen, C. Y. Chiu, L. Ruan, Y. Liu, M. Li, X. Duan and Y. Huang, Nano Lett., 2014, 14, 3887-3894.
39 Y. Wang, S.-I. Choi, X. Zhao, S. Xie, H.-C. Peng, M. Chi, C. Z. Huang and Y. Xia, Adv. Funct. Mater., 2014, 24, 131-139. 40 R. Blume, H. Niehus, H. Conrad and A. Bottcher, J. Phys. Chem. B, 2004, 108, 14332-14339.

41 X. Huang, Z. Zhao, L. Cao, Y. Chen, E. Zhu, Z. Lin, M. Li, A. Yan, A. Zettl, Y. M. Wang, X. Duan, T. Mueller and Y. Huang, Science, 2015, 348, 1230-1234.

42 Z. Zhang, Y. Ji, J. Li, Z. Zhong and F. Su, RSC Adv., 2015, 5, 54364-54371. 